\title{
Effects of food primes on the operant behavior of nondeprived rats
}

\author{
LEONARD A. EISERER \\ Franklin and Marshall College, Lancaster, Pennsylvania 17604
}

\begin{abstract}
After rats had been trained to press a lever for food reward, experimenter-initiated food "primes" increased the likelihood of subsequent responding during periods in which the subjects were nondeprived. No such priming effects were found after presentation of a stimulus that had previously been paired with food. In other experiments, nonreinforced leverpresses, as well as subthreshold components of the leverpress response (e.g., forepaw raising), were also found to be enhanced by food primes. Taken together with other reports in the literature, the present findings are consistent with a "motivational aftereffects" interpretation of priming, and also with the notion that all stimuli which possess reinforcing properties possess priming properties as well.
\end{abstract}

Although the term "priming effect" can have several different meanings within psychology (e.g., Neely, 1977), it often refers to response enhancement that follows the noncontingent (i.e., response-independent) presentation of a reinforcing stimulus. This type of effect has been repeatedly demonstrated in studies involving positively reinforcing brain stimulation (Deutsch \& Howarth, 1963; Gallistel, 1964; Old's, 1955). For example, rats trained to press a lever for brain stimulation reward can readily be induced (by an experimenter-induced brain stimulus) to respond during periods when leverpresses would otherwise be unlikely to occur (e.g., during extinction).

Comparable priming effects can also be readily demonstrated in the context of imprinting (Eiserer, 1977; Eiserer \& Hoffman, 1973). Thus, ducklings trained to peck a pole for the rewarding presentation of an imprinting object can be induced, via a brief stimulus presentation, to respond during periods when they are otherwise little inclined to respond. As with brain stimulation, priming effects with an imprinting object are generally robust and easy to demonstrate.

Apparently more elusive, however, are priming effects with such conventional reinforcers as food and water since, as noted by Gallistel (1973), it is "difficult to find priming-like effects in the literature on natural rewards" (p. 195). Much has been made of this fact in the past; indeed, it has been asserted that priming effects represent one of several phenomena which indicate the existence of fundamental differences between brain stimulation and an imprinting object, on the one hand, and conventional reinforcers, on the other (regarding brain stimulation, see Gallistel, 1973; Trowill,

Requests for reprints should be sent to the author at Whitely Psychology Laboratories, Franklin and Marshall College, Lancaster, Pennsylvania 17604.
Panksepp, \& Gandelman, 1969; regarding imprinting, see Eiserer \& Hoffman, 1973).

And yet, several priming-like effects have in fact been demonstrated with conventional rewards, although the available literature is admittedly scattered and sometimes only incidentally related to priming per se. For example, in early work that was actually directed at other issues, Bruce (1937) reported "a characteristic increase in locomotion (in a runway) when a small amount of water is given the animals, if compared to the speed of running after approximately the same amount of practice without initial water." [Gallistel (1973), it should be noted, has contested Bruce's conclusion.] And, in essentially a replication of Bruce's design, Morgan and Fields (1938) found that small amounts of prefeeding can increase locomotor speed in at least the early segments of a runway.

In reporting the work of other researchers, Konorski (1967) noted that a dog "was trained to perform two different movements: one for procuring bread, and the other for procuring water." Priming the dog with either food or water "unmistakably led to the performance of the food movement or the water movement respectively" (p. 21). In another study, Panksepp and Trowill (1967) trained rats to press a lever for chocolate milk reward and found that, after responding had subsequently ceased during extinction procedures, milk primes reinstated leverpressing in the majority of cases. Even more recently, Deluty (1976) reported that food delivered independently of rats' behavior produced excitatory aftereffects with respect to a previously reinforced leverpress response.

The present series of experiments was designed to further demonstrate and examine priming effects with food in the context of an operant response. In doing so, the work employed rats which, except for an initial period of response shaping, were not deprived of food. This insured a low response baseline against which any enhancement effects of primes could be readily observed. 


\section{EXPERIMENT 1}

In addition to assessing the priming properties of food, the first experiment also sought to determine whether a stimulus that had previously been associated with food would itself produce "conditioned" priming effects. The conditioned stimulus that was used for this purpose was the "click" sound that occurred whenever the food magazine was operated.

\section{Method}

Subjects. Four male albino rats served as subjects. Except for 4 days of leverpress training during which the animals were on a 12-h food-deprivation schedule, all rats had continuous access to Agway laboratory chow as well as water in their home cages.

Apparatus. The operant conditioning chamber $(23 \times 20 \times$ $20 \mathrm{~cm}$ ) contained a lever mounted on the intelligence panel, approximately $7 \mathrm{~cm}$ above the floor and $7.5 \mathrm{~cm}$ from either side of the chamber. The food magazine, through which $45-\mathrm{mg}$ standard formula Noyes pellets could be delivered, was located in the bottom left corner of the intelligence panel. A water bottle was mounted on the panel directly opposite the lever. Standard recording equipment was used to monitor the rats' leverpress responses.

The present study required that the experimenter be able to present occasionally the clicking sound that the magazine made during its operation, without also presenting any accompanying food. This stimulus condition was accomplished by activating a tape recorder upon which the sound of the operating magazine had previously been recorded (the recorder could be operated in such a way that turning the machine on or off did not in itself produce any appreciable sound). In response to two separate test presentations of the click during the latter half of initial shaping procedures, each of the rats went over to the food tray and sniffed inside.

Procedure. After the rats were trained to leverpress with Noyes pellets as reinforcement, the 12-h deprivation schedule was changed to an ad-lib feeding schedule in the home cages. Each subject was then given four experimental sessions during which every leverpress was reinforced with presentation of one pellet.

At the start of each session, a subject was placed in the experimental chamber and permitted to initiate responding on its own. The response rate was then monitored so that whenever the rat permitted 2 min to pass without a leverpress, one of three events occurred: a pellet was delivered into the food tray (a prime trial); the sound of the magazine in operation was presented without any accompanying food (a click trial); or no stimulus change occurred (a baseline trial). Following a given event, the criterion of no responses for $2 \mathrm{~min}$ had again to be met before the next trial began.

The three types of trials occurred in random order, and each session terminated when four trials of each type had been completed. One such session was run on each of 4 successive days.

\section{Results}

Throughout the present experiment, as well as throughout the two subsequent studies, no rat ever discovered (i.e., sniffed) a pellet in the food tray without also immediately eating it.

At the start of each session in the present experiment, the rats typically spent a minute or so moving and sniffing around the chamber before finally effecting an initial leverpress. They then usually emitted a series of responses (collecting the pellet after each press), after which their activity level declined noticeably. Indeed, the rats often yawned, stretched, groomed, or simply curled up and rested. Periodically, however, they would move about the chamber, perhaps sniff inside the food tray (a behavior all the rats performed more or less regularly), or emit a brief series of responses.

Despite the immediate reaction that the rats gave during initial shaping procedures whenever the sound of the magazine was presented (whether the actual sound or the taped recording), they characteristically showed little more than a minor orienting response (ear twitching, head raising, etc.) when the sound was presented during the course of the actual experiment. Even on prime trials, some amount of time typically elapsed before a rat, usually in the course of his periodic movements about the chamber, came upon the pellet in the food tray.

Priming effects were assessed in terms of the probability of response initiation in the 1 -min interval immediately following either presentation of the prime, presentation of the click, or the corresponding absence of stimulus presentation on baseline trials. These probabilities, presented for each rat in Table 1, represent the proportion of 1-min measurement periods (a total of 16 under each condition) that contained one or more responses.

As can be seen from the table, all of the rats showed greater probability of response initiation during prime trials than during either click or baseline trials, while the differences between the latter two types of trials were inconsistent. An analysis of variance for repeated measures confirmed the reliability of the priming effect $[F(2,6)=23.15, p<.01]$.

Inspection of the data on a session-by-session basis failed to reveal any trend of change in the probabilities.

\section{Discussion}

The fact that the rats always ate the Noyes pellets even though they had not been deprived of laboratory chow in their home cages suggests that the pellets were more palatable than the chow. More surprising, perhaps, was the finding that the rats did not react to the magazine click during the experimental sessions. This was apparently related to the subjects' being nondeprived, since deprivation level was the primary difference between the conditions of the initial shaping (when they

Table 1

Response-Initiation Probabilities for Individual Subjects on Prime, Click, and Baseline Trials

\begin{tabular}{cccc}
\hline Subject & Prime & Click & Baseline \\
\hline 1 & .19 & .00 & .06 \\
2 & .56 & .13 & .19 \\
3 & .63 & .38 & .31 \\
4 & .38 & .13 & .06 \\
Mean & .44 & .16 & .16 \\
\hline
\end{tabular}


did react to the click) and those of the experimental sessions.

Consistent with this inference are reports that the secondary or conditioned reinforcing properties of stimuli previously paired with conventional rewards appear to be weak or nonexistent for nondeprived subjects (Estes, 1949; Schlosberg \& Pratt, 1956). Interestingly, conditioned reinforcement has also been difficult to demonstrate with brain stimulation reward, a difficulty that has been attributed to the fact that most studies involving brain stimulation utilize nondeprived subjects (Lenzer, 1972; Trowill, Panksepp, \& Gandelman, 1969). Thus, regarding the present lack of priming by the magazine click, it is possible that conditioned priming properties, like conditioned reinforcing properties, depend upon some degree of deprivation for their ability to affect behavior.

The present study, in any event, has demonstrated reliable priming effects with food. Since priming effects have also been found with milk (Panksepp \& Trowill, 1967), brain stimulation (Deutsch \& Howarth, 1963), and an imprinting object (Eiserer \& Hoffman, 1973), one might speculate that all stimuli which possess reinforcing properties may possess priming properties as well.

\section{EXPERIMENT 2}

While Experiment 1 involved responses that were always reinforced, studies with both brain stimulation and an imprinting object have shown that primes with those stimuli are able to facilitate even responses undergoing extinction (Deutsch \& Howarth, 1963; Eiserer \& Hoffman, 1973). The question thus arises as to whether food primes can have the same effect. Experiment 2 investigated this issue by assessing priming effects on leverpressing during periods when such behavior was not reinforced.

In doing so, the present study also attempted to avoid a difficulty that arose from the procedure used in Experiment 1; namely, the irregular time lag occurring between presentation of primes and the rats' actual discovery of the pellets in the food tray. To circumvent this problem, the present study required an additional behavioral criterion before initiation of either a prime or a baseline trial, as described below.

\section{Method}

Subjects and Apparatus. The same subjects and apparatus used in Experiment 1 were employed in Experiment 2.

Procedure Each rat received eight experimental sessions at the rate of one session per day. At the start of these sessions, each of the first 10 responses was reinforced with a single food pellet. However, the 11 th response was not reinforced and marked the beginning of a period of extinction in which successive leverpresses were also not reinforced. Prime and baseline trials were then initiated in random order as the subject met both of the following criteria: (1) emission of no responses for a $2-\min$ period, and (2) having met this criterion, the rat stuck its nose into the empty food tray (all of the rats showed this latter behavior every now and then during the course of moving about the chamber). As soon as these criteria were reached, the rat either received an experimenter-delivered food pellet (a prime trial) or he did not (a baseline trial).

Extinction conditions terminated $1 \mathrm{~min}$ after the occurrence of a given prime or baseline event, whereupon the rat's next 10 responses were again reinforced by single food pellets. The 11 th response was not reinforced and marked the start of another extinction period during which either a prime or baseline trial occurred. The session ended when two trials of each type had been completed.

\section{Results and Discussion}

Shown in Table 2 are the probabilities of response initiation during the $1-\mathrm{min}$ interval following prime and baseline events. As can be seen from the table, all of the rats showed greater probability of response initiation during prime trials than during baseline trials; this difference was statistically significant $[\mathrm{t}(3)=3.90$, $\mathrm{p}<.05]$.

Inspection of Tables 1 and 2 reveals that all of the response probabilities obtained in the present experiment were higher than the corresponding probabilities obtained in Experiment 1. This increase could have resulted from the trial criteria used in Experiment 2, since those criteria insured that each rat was near the food tray (and hence near the lever) at the start of each trial. This is only one possible explanation, however, since other procedural differences also existed between the two studies (e.g., the use of more experienced subjects in Experiment 2, and of course the extinction manipulation itself).

In any event, the present study has demonstrated that primes with food (like primes with brain stimulation and an imprinting object) are capable of facilitating operant behavior even when that behavior fails to produce the reinforcing stimulus.

\section{EXPERIMENT 3}

In Experiment 1, leverpresses followed food primes on $43 \%$ of the prime trials. While this might imply that the primes produced no effects on $57 \%$ of the trials, it is also possible that their influence was often merely subthreshold for initiation of leverpresses. This latter possibility was assessed in Experiment 3 by monitoring the rats' behavior on those trials (both prime and base-

Table 2

Probabilities of Response-Initiation on Prime and Baseline Trials During Periods of Extinction

\begin{tabular}{ccc}
\hline Subject & Prime & Baseline \\
\hline 1 & .94 & .25 \\
2 & .69 & .44 \\
3 & .69 & .44 \\
4 & .75 & .31 \\
Mean & .77 & .41 \\
\hline
\end{tabular}


line) in which no leverpresses occurred. More specifically, the study sought to determine whether certain subthreshold components of leverpressing (namely, a rat's turning in the direction of the lever and then lifting his forepaws) were facilitated by primes on those trials where leverpressing itself did not actually occur.

\section{Method}

Subjects and Apparatus. The same subjects and apparatus used in Experiments 1 and 2 were used in Experiment 3.

Procedure. Each rat received six experimental sessions at the rate of one session per day. During these sessions, whenever the rat (1) permitted 2 min to pass without a response, and (2) stuck its nose into the empty food tray, either a food pellet was delivered into the tray (a prime trial) or it was not (a baseline trial). Following a given event, the above-described criteria had again to be met before the next trial occurred.

The two types of trials occurred in random order, and each of the six sessions terminated when four trials of each type had been completed. Throughout these sessions, all of the subjects' behavior was under the continuous observation of the experimenter.

\section{Results}

The present data were analyzed by computing two different response probabilities: (1) the probability of occurrence of at least one leverpress within the 1-min interval immediately following initiation of either a prime or a baseline trial; and (2) the probability that, on those trials in which a leverpress did not occur, the rat first turned in the direction of the lever and then reared up (i.e., lifted both forepaws off the floor of the experimental chamber). The results of these computations are shown in Table 3 .

As can be seen from the table, all of the rats showed a greater probability of leverpressing on prime trials than on baseline trials $[t(3)=4.83, p<.05]$. Moreover, on those trials where no leverpresses were emitted, all of the rats showed a greater probability of turning and rearing towards the lever on prime trials than on baseline trials $[\mathrm{t}(3)=5.53, \mathrm{p}<.05]$.

\section{Discussion}

Even on trials where the effects of a food prime were insufficient to induce a complete leverpress response, certain components of that response were nevertheless significantly enhanced. The present study thus offers

Table 3

Probabilities of Leverpressing on Prime and Baseline Trials, and Probabilities of Turning and Rearing on Those Trials Where Leverpressing Did Not Occur

\begin{tabular}{cccccc}
\hline & \multicolumn{2}{c}{ Leverpress } & & \multicolumn{2}{c}{ Turn and Rear } \\
\cline { 2 - 2 } \cline { 5 - 6 } Subject & Prime & Baseline & & Prime & Baseline \\
\hline 1 & .21 & .04 & .63 & .35 \\
2 & .46 & .29 & .23 & .12 \\
3 & .63 & .42 & .44 & .14 \\
4 & .58 & .21 & .40 & .11 \\
Mean & .47 & .24 & .43 & .18 \\
\hline
\end{tabular}

empirical confirmation for a supposition that doubtless many researchers take for granted -namely, that a precisely defined operant response (such as a leverpress) should not be considered an all-or-none event but rather the culmination of a graduated series of constituent behaviors. These behaviors can be assessed separately and may actually be, as in the present case, more sensitive to the operations of an independent variable than is the defined operant response.

Although the rats as a group leverpressed on $47 \%$ of the prime trials, this percentage increases to $71 \%$ if one considers those trials on which at least turning and rearing (but not necessarily leverpressing) occurred. Perhaps if priming effects had been assessed in terms of behavior even more elementary than turning and rearing, or at perhaps the neural level (e.g., in the brainstem arousal system; Rolls, 1971), then primes may have been found to affect the rats on virtually $100 \%$ of the trials.

\section{GENERAL DISCUSSION}

Except for numerous studies within the brain stimulation literature, priming effects have not received a great deal of experimental attention. This lack is somewhat surprising, especially since priming-like effects are seemingly a matter of common experience in everyday life. Indeed, priming has aptly been referred to as the "salted peanut" phenomenon (Konorski, 1967, p. 21) in apparent acknowledgement of its manifestation at cocktail parties and the like. As Hebb (1949) has observed: "Ordinarily, one can take salted nuts or let them alone-until one has eaten a mouthful, when it becomes much harder to let them alone" (p. 199).

This conception of priming implies a particular interpretation regarding its mode of operation-namely, that primes leave motivational aftereffects which induce organisms to seek additional stimulation of the same sort. A comparable motivational view has been offered as a possible explanation for priming effects demonstrated in the laboratory with both brain stimulation and an imprinting object (Deutsch \& Howarth, 1963; Eiserer \& Hoffman, 1973). Importantly, strong support for this motivational hypothesis has been provided by studies showing a fair amount of specificity in priming effects when, for example, brain stimulation is pitted against water reward (Deutsch, Adams, \& Metzner, 1964) or when water is pitted against food (Konorski, 1967).

In conceptualizing priming effects experimentally, it is useful to compare them with reinforcement (i.e., positive reinforcement) effects. Both priming and reinforcement involve an enhancement of a specifiable response, an enhancement that depends upon presentation of some positively valenced stimulus (food, an imprinting object, etc.). However, the enhancement in the case of reinforcement generally represents a rela- 
tively long-termed learning effect, while the enhancement in priming probably reflects a short-termed motivational effect.

Procedurally, priming and reinforcement are two very different experimental events. In the reinforcement paradigm, the response (such as a leverpress) must generally be emitted before presentation of the valenced stimulus if it is to be facilitated, while in the priming procedure the response is enhanced even if it does not occur before the stimulus (as in the present work). Another difference involves the role that temporal contiguity plays in the two procedures. In reinforcement, the contiguity existing between the valenced stimulus and the response that preceded it is usually crucial. In priming, however, the important contiguity is that which exists between the valenced stimulus and the subsequently emitted response; indeed, priming effects are severely weakened if the opportunity to emit the primed response is delayed after stimulus presentation (Howarth \& Deutsch, 1962; Morgan \& Fields, 1938).

Still another difference between priming and reinforcement is that although the specified response can be almost any behavior whatsoever in the reinforcement paradigm, the enhanced response in the priming procedure is best conceived as having some preexisting association (whether learned or innate) with the valenced stimulus. In the present work, for example, leverpressing presumably could not have been primed with food had that response not earlier been associated with food (in this case, via shaping procedures).

\section{REFERENCES}

BRUCE, R. H. An experimental investigation of the thirst drive in rats with especial reference to the goal-gradient hypothesis. Journal of General Psychology, 1937, 17, 49-60.

DELUTY, M. Z. Excitatory and inhibitory effects of free reinforcers. Animal Learning \& Behavior, 1976, 4, 436-440.

Deutsch, J. A., Adams, D. W., \& Metzner, R. J. Choice of intracranial stimulation as a function of delay between stimulations and strength of competing drive. Journal of Comparative and Physiological Psychology, 1964, 57, 241-243.

Deutsch. J. A., \& Howarth, C. I. Some tests of a theory of intracranial self-stimulation. Psychological Review, 1963, 70. 444-460.

EISERER. L. A. Behavioral control by stimulus components of an imprinting object. Animal Learning \& Behavior, 1977, 5, 153.160.

Eiserer, L. A., \& Hoffman, H. S. Priming of ducklings' responses by presenting an imprinted stimulus. Journal of Comparative and Physiological Psychology, 1973, 82, 345-359.

Estes. W. K. A study of motivating conditions necessary for secondary reinforcement. Journal of Experimental Psychology, 1949, 39, 306-310.

Gallistel, C. R. Electrical self-stimulation and its theoretical implications. Psychological Bulletin, 1964, 61, 23-34.

Gallistel. C. R. Self-stimulation: The neurophysiology of reward and motivation. In J. A. Deutsch (Ed.), The physiological basis of memory. New York: Academic Press, 1973.

HEBB, D. O. The organization of behavior. New York: Wiley, 1949.

Howarth, C. I., \& Deutsch, J. A. Drive decay: The cause of fast "extinction" of habits learned for brain stimulation. Science. 1962, 137. 35.36.

KONORSK1, J. Integrative activity of the brain. Chicago: University of Chicago Press. 1967.

LENZER, I. I. Differences between behavior reinforced by electrical stimulation of the brain and conventionally reinforced behavior: An associative analysis. Psychological Bulletin, 1972, 78, 103-118.

Morgan. C. T., \& Fields, P. E. The effect of variable preliminary feeding upon the rat's speed-of-locomotion. Journal of Comparative Psychology, 1938, 26, 331-348.

Neely, J. H. Semantic priming and retrieval from lexical memory: Roles of inhibitionless spreading activation and limited-capacity attention. Journal of Experimental Psychology: General. 1977. 106. 226-254.

Olds, J. Physiological mechanisms of reward. In Nebraska symposium on motivation (Vol. 3). Lincoln: University of Nebraska Press, 1955. Pp. 73-139.

Panksepp, J., \& Trowill, J. A. Intraoral self injection: The simulation of self-stimulation phenomena with a conventional reward. Psychonomic Science, 1967, 9. 407.408.

RolLs, E. T. Involvement of brainstem units in medial forebrain bundle self-stimulation. Physiology and Behavior, 1971, 7, 297-310.

Schlosberg, H., \& Pratt, C. The secondary reward value of inaccessible food for hungry and satiated rats. Journal of Comparative and Physiological Psychology, 1956, 49, 149-152.

Trowill, J. A., Panksepp, J., \& Gandelman, R. An incentive model for rewarding brain stimulation. Psychological Review, 1969, 76, 264-281.

(Received for publication July 26, 1977; revision accepted January 23,1978 .) 